\title{
Avaliação das Estratégias de Autoaprendizagem em Alunos de um Curso de Medicina em Belém - Pará
}

\section{Evaluation of Self-Directed Learning Strategies in Students of a Medicine Course in Belém - Pará}

\author{
Irma Douglas Paes Barreto ${ }^{\text {e }}$ \\ Patrick Abdala Gomes \\ Ismari Perini Furlaneto ${ }^{I}$ \\ Bruno Barreto ${ }^{I, I}$
}

\section{PALAVRAS-CHAVE}

- Aprendizagem.

- Aprendizagem Baseada em Problemas.

- Estratégias.

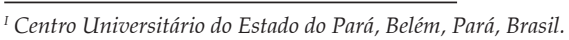

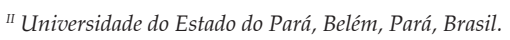

\begin{abstract}
RESUMO
Introdução: A Aprendizagem Baseada em Problemas (ABP) é uma metodologia de ensino-aprendizagem que tem sido incorporada aos currículos das escolas médicas que priorizam o estudante no centro da discussão. O diagnóstico e a mensuração das estratégias de estudo e aprendizagem constituem um dos aspectos fundamentais à melhoria do processo de ensino-aprendizagem. Objetivo: O estudo teve como objetivo identificar as estratégias do discente em desenvolver seu estudo autodirigido, avaliando a utilização de ferramentas úteis no contexto do seu ensino-aprendizagem. Metodologia: Foi realizado um estudo transversal observacional descritivo e analítico, aplicando-se 348 questionários a alunos de um curso de Medicina, uniformemente distribuídos do segundo ao nono semestres. Os questionários, do tipo Likert, foram direcionados para investigar as estratégias utilizadas no estudo individual e sua percepção desses alunos em relação à aprendizagem. A análise estatística foi feita por diferentes métodos, de acordo com as variáveis e categorias observadas, e executada com auxílio do software BioEstat 5.45. Resultados com $p \leq 0,05$ (bilateral) foram considerados significativos. Resultados: A média de idade foi de 22,7 anos, com 57,3\% de mulheres. A maioria mostrou hábito de estudo superior a três vezes por semana (72,2\%), em lugares fixos, sobretudo na biblioteca (96,8\%). A maioria dos alunos dispunha de recursos eletrônicos para acessar a internet. A maioria dos respondentes $(43,6 \%)$ utilizou muito pouco o recurso de videoaula. Cinquenta por cento dos alunos julgaram fácil cumprir o horário de estudo e evitaram situações que desviavam sua atenção. Cinquenta por cento deles estudaram sozinhos todos os objetivos e mantiveram foco nos mesmos. O uso do mapa conceitual não foi frequente, mas a maioria elaborou esquemas durante o estudo. Para a maioria dos estudantes, a busca de artigos científicos no idioma inglês aconteceu apenas em $50 \%$ das vezes. Apenas $25 \%$ dos alunos fizeram "sempre" a síntese do estudo em forma de resumo. No mínimo $25 \%$ dos estudantes utilizaram mais de um livro-texto em seus estudos, e a acesso a artigos por meio de plataformas de bases de dados científicos ocorreu "sempre". Em relação ao uso de apostilas comerciais e resumos não próprios, até $75 \%$ dos alunos responderam que isso ocorre em aproximadamente " $50 \%$ das vezes". A autopercepção da retenção do conhecimento, para $50 \%$ dos estudantes, ocorreu "cerca de $50 \%$ das vezes" ou com "muita frequência". Conclusão: Alunos deste estudo que trabalham com a metodologia ABP utilizaram recursos apropriados para seu estudo, planejando e organizando sua aprendizagem. O hábito de planejar o estudo esteve positivamente associado às melhores estratégias de aprendizagem eà busca por fontes de melhor qualidade técnica, resultando em maior percepção da retenção do conhecimento pelo aluno. Como houve falhas nas estratégias de aprendizagem, sugere-se a criação de um guia de orientações de autoaprendizagem para auxiliar o aluno no desempenho de seu estudo.
\end{abstract}




\section{KEY-WORDS}

- Learning.

- Problem-Based Learning.

- Strategies.

\section{ABSTRACT}

Introduction: Problem-Based Learning $(P B L)$ is a teaching and learning methodology that has been incorporated into the curricula of medical schools, which prioritize the student to the center of the discussion. The diagnosis and measurement of learning strategies has become a key aspect in the improvement of the teaching and learning process. Objective: The study aims to identify students' strategies in developing their self-directed learning, evaluating the application of useful tools in the context of their learning. Methods: A descriptive and analytical observational cross-sectional study was carried out, applying 348 questionnaires to the students of a medical school, uniformly distributed from the $2^{\text {nd }}$ to $9^{\text {th }}$ semesters of the course. The Likert-scaled questionnaires were aimed at investigating the strategies used for individual study as well as student perception regarding learning. Statistical analysis was performed by different methods, according to the variables and categories observed and executed with the aid of BioStat 5.45 software. Results with $p \leq 0.05$ (bilateral) were considered significant. Results: The mean age was 22.7 years, with $57.3 \%$ being female. The majority showed a study habit higher than three times a week $(72.2 \%)$, in fixed places, especially in the library (96.8\%). Most of the students have electronic resources to access the internet. Most of the respondents (43.6\%) use the video-lecture feature infrequently. 50\% of the students find it easy to follow scheduled study hours and avoid situations that distract their attention. 50\% of them studied all proposed learning objectives alone, and kept their focus. The use of the conceptual map is not frequent. However, most developed schemes during study. The majority of the students conducted searches for scientific articles in the English language only 50\% of the time. Only 25\% of the students «always» synthesize the study in summary form. At least $25 \%$ of the students used more than one textbook in their studies and 'always' accessed articles through scientific database platforms. Regarding the use of commercial e-books and nonself summaries, up to $75 \%$ of the students answered that this happens approximately $50 \%$ of the time. Regarding self-perception of knowledge retention, for $50 \%$ of the students the answers varied between this happens "about $50 \%$ of the time» and "very frequently". Conclusion: The medical students using PBL methodology, use appropriate resources for their study, planning and organizing their learning, however there have been failures in learning strategies. A habit of planning study is positively associated with the best learning strategies and the search for sources of better technical quality, resulting in a greater perception of student retention of knowledge. Therefore, it is suggested that self-learning guidelines be created to assist the student in his or her study performance.

Recebido em: 5/3/19

Aceito em: 1/4/19

\section{INTRODUÇÃO}

Os avanços da medicina e o compromisso da graduação com uma formação adequada são desafios que têm como objetivo fundamental possibilitar ao futuro médico uma base sobre a qual ele possa se desenvolver e aprender continuamente.

Proporcionar estratégias para ensinar aos estudantes de Medicina como aprender é difícil, visto que chegam à universidade cada um com sua história, etnia e cultura, oriundos de diversas instituições, carregando consigo diferentes estilos de aprendizagem $^{1,2}$.

Surge, então, a necessidade de repensar o modo de ensinar e aprender em um mundo com novas tecnologias e rapidez na circulação de informações. A capacitação docente na formação médica, a atualização profissional permanente e as mudanças das políticas educacionais convergem para este novo modelo educacional ${ }^{3,4}$.

As Diretrizes Curriculares Nacionais (DCN) inserem uma seção para o curso de Medicina, que trata especificamente de Educação em Saúde e Educação Permanente, segundo a qual o graduando deverá se corresponsabilizar pela própria formação inicial e permanente, conquistando progressivamente a autonomia intelectual ${ }^{5}$.

Neste sentido, acompanhando a explosão científica observada em todos os campos do conhecimento, metodologias ativas de ensino e aprendizagem, como a Aprendizagem Baseada em Problemas (ABP), têm sido incorporadas aos currículos das escolas médicas. Estas, no entanto, não mudaram a duração de seus cursos, o que torna insuficiente o tempo 
disponível para ensinar todo o corpo do conhecimento médico curricular necessário ${ }^{1,3,4}$.

Desta forma, o modelo de ABP mostrou-se real e efetivo para a educação médica, já que se baseia nas modernas teorias de aprendizagem, caracterizando-se principalmente por colocar o estudante no centro do processo, desenvolvendo as capacidades de construir ativamente o próprio aprendizado. Na Região Norte, o Centro Universitário do Estado do Pará (Cesupa) foi pioneiro neste modelo ${ }^{6,7}$.

O processo de autoaprendizagem é uma iniciativa individual, com ou sem ajuda de outros (alunos e/ou tutores), cujo objetivo seria diagnosticar as próprias necessidades de aprendizagem $^{8,9}$. Já as estratégias de aprendizagem são técnicas ou métodos que os alunos usam para adquirir a informação, por meio de sequências de procedimentos ou atitudes escolhidos com a finalidade de facilitar a aquisição, o armazenamento e/ ou a utilização da informação ${ }^{10.11}$.

As estratégias de estudo e aprendizagem adotadas por alunos de cursos superiores têm-se mostrado eficientes para uma aprendizagem mais significativa, exigindo do aluno organização de espaços e tempo para a realização das tarefas propostas, além da habilidade para acessar e identificar pesquisas bibliográficas de relevância e capacidade de análise crítica dessas informações. Ressalte-se a eficácia de tais métodos em diminuir as dificuldades pessoais dos alunos, engrandecer a aprendizagem e controlar melhor os fatores ambientais que interferem em um bom desempenho no estudo ${ }^{9-12}$. O diagnóstico, a mensuração e o estudo dessas estratégias constituem um dos aspectos fundamentais para a melhoria do processo de ensino-aprendizagem, justamente por possibilitar a implementação de programas de orientação quanto aos hábitos de estudo ${ }^{13,14}$.

Desta maneira, por meio das mudanças das metodologias de ensino-aprendizagem, explosão de conhecimentos científicos, fácil acesso a novas tecnologias e propostas de egressos baseadas na educação continuada, tem-se como objetivo neste estudo avaliar as estratégias de autoaprendizagem utilizadas, desenvolvidas e referidas pelos estudantes de um curso de Medicina em Belém, no Pará.

\section{MÉTODO}

\section{Aspectos éticos}

O presente projeto foi aprovado pelo Comitê de Ética do Centro Universitário do Estado do Pará-Cesupa sob o número 1.704.621.

\section{Tipo de estudo}

Trata-se de um estudo transversal de natureza observacional exploratória e de caráter descritivo e analítico, precedido de um estudo piloto que investigou as possíveis dificuldades na compreensão do instrumento.

\section{Amostra e amostragem}

Foram incluídos alunos de ambos os sexos, com idade igual ou superior a 18 anos, regularmente matriculados entre o segundo e o nono semestre do curso de Medicina do Cesupa e que estão cursando ou já cursaram os módulos tutoriais baseados no método de ABP, entre fevereiro e março de 2017.

\section{Critérios de inclusão e exclusão}

Foram excluídos das análises os questionários que não continham respostas para no mínimo $67 \%$ dos itens de cada domínio investigado e/ou que tenham deixado de responder a quaisquer perguntas que caracterizassem o perfil do participante.

\section{Instrumento e coleta de dados}

Foi utilizado um questionário semiestruturado elaborado pelos próprios autores, o qual continha perguntas (itens) que investigaram alguns aspectos do hábito e das estratégias de estudo dos alunos, distribuídos em quatro domínios.

Os aspectos abordados foram explorados sempre por meio da identificação da frequência com que o aluno executava a atividade ou recorria às estratégias de aprendizagem expostas sob a forma de afirmativas, cujas alternativas de respostas correspondiam a uma escala ordinal do tipo Likert. Com exceção de dois itens que precisaram ser recodificados, as alternativas estavam ordenadas em ordem crescente, de forma que maiores escores (maiores frequências) correspondiam ao uso de melhores estratégias e/ou recursos de aprendizagem utilizados. Além disso, foi incluída a opção "não desejo responder". Também foram coletadas informações que caracterizassem o perfil sociodemográfico e econômico dos respondentes.

Os questionários foram distribuídos para que, após exposição dos objetivos do estudo, fossem respondidos pelos próprios acadêmicos, em momento exclusivo e individualmente.

O fluxo metodológico empregado nesta pesquisa está representado na Figura 1.

\section{Definição dos domínios investigados}

Os itens foram avaliados por cinco juízes, todos docentes pesquisadores em educação médica, que os agruparam nos seguintes domínios: "planejamento e organização do estudo", que considerou a capacidade do aluno para planejar e seguir uma rotina que promova um ambiente favorável a seus estudos; "estratégias de aprendizagem", definido como o uso de métodos elaborados e utilizados para otimizar o tempo e 


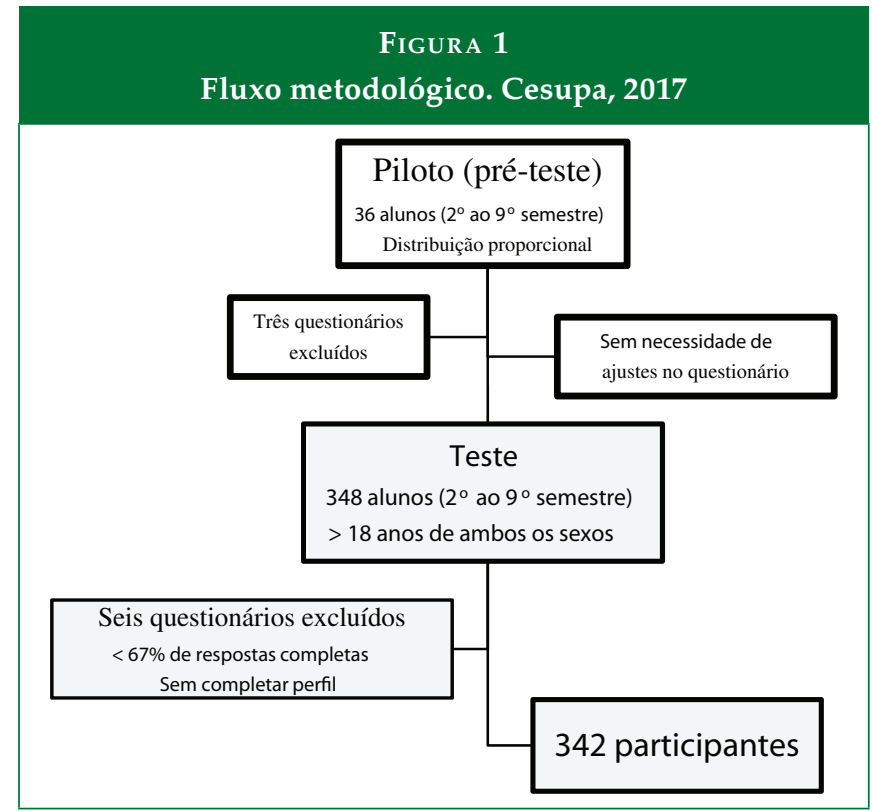

promover memorização; "qualidade das informações" pesquisadas, compreendida como a confiabilidade das fontes utilizadas para o estudo; e "autopercepção da retenção do conhecimento", que incluiu itens que abordavam o próprio julgamento quanto à assimilação dos conteúdos apreendidos em curto e médio prazos.

\section{ANÁLISE DOS DADOS}

Os dados referentes às características sociodemográficas e econômicas foram tratados por meio de estatística descritiva, expressos sob a forma de média \pm desvio padrão, mediana e percentis e frequências absoluta e relativa, conforme o caso.

As respostas a cada item Likert, expressas sob a forma de uma afirmação e pertencentes a um de cinco domínios, foram agrupadas de acordo com o domínio correspondente, possibilitando a avaliação entre domínios.

O escore total de cada domínio consistiu no produto da média aritmética simples dos escores das questões que o compõem pelo total de questões do domínio, de forma que fosse possível compensar a nulidade de até 33\% dos itens não respondidos, resultando em um escore final entre 2 e 10 para domínios com dois itens, 3 e 15 para domínios com três itens, 5 e 25 para domínios com cinco itens, e 7 e 35 para aqueles com sete itens.

Foi construída uma sintaxe que foi inserida numa planilha do software Microsoft Excel ${ }^{\circledR}$, que permitiu calcular o escore de cada domínio e convertê-lo numa escala de 0 a 100. Quanto maior o escore obtido, mais frequente e, consequentemente, melhor será a forma de executar determinada atividade.
A comparação de um mesmo domínio entre as diferentes turmas foi feita com o auxílio de Análise de Variância não paramétrica de Kruskal-Wallis, e a comparação dos diferentes domínios dentro da mesma turma foi feita pela Análise de Variância de Friedman. A Análise de Variância paramétrica (um critério), seguida pelo teste de Tukey (no caso de significância), foi utilizada para comparar médias de amostras independentes.

O Qui-Quadrado ou Teste G de aderência foi empregado para comparar proporções de categorias dentro de uma mesma amostra.

A Correlação de Spearman foi utilizada para estimar a correlação entre a estratégia de planejamento e organização do estudo e a autopercepção da retenção do conhecimento, e entre esta e as estratégias de aprendizagem.

Foram considerados "desejáveis" escores medianos $\geq 4$, correspondentes a uma escala centesimal igual a 75\%, indicando que tal atitude é executada com frequência superior a 50\%.

Todos os testes foram executados pelo software BioEstat $5.4^{15}$, e resultados com $p \leq 0,05$ (bilateral) foram considerados significativos.

\section{RESULTADOS E DISCUSSÃO}

\section{Perfil sociodemográfico}

Foram aplicados, aleatoriamente, questionários a 348 discentes de oito turmas do curso de Medicina, dos quais seis alunos $(1,7 \%)$ foram excluídos. Os participantes estavam uniformemente distribuídos entre o segundo e o nono período do curso $(\mathrm{p}=0,4846)$.

A média de idade dos participantes foi igual a $22,7 \pm 4,3$ anos (IC 95\%: 22,2 - 23,1), variando entre 18 e 49 anos. Esta média caracterizou alunos da geração $Z$, nascida, em grande maioria, após a década de 1990, como esperado de alunos mais jovens nos primeiros semestres.

Houve maior proporção de mulheres entre os 342 respondentes $(57,3 \%$ vs. $42,7 \%$; $\mathrm{p}=0,0081)$, porém a proporção de indivíduos dos sexos masculino e feminino foi semelhante entre as turmas ( $\mathrm{p}=0,9344)$.

A maioria dos participantes $(98,0 \%, 335 / 342 ; p<0,0001)$ cursou o ensino médio em instituições particulares, não possui graduação prévia $(90,7 \%, 310 / 342 ; p<0,0001)$ e não exerce atividade remunerada $(94,2 \%, 322 / 342 ; p<0,0001)$.

\section{Modo preferencial de acesso às informações}

Quanto ao uso da biblioteca, 96,8\% (331/342; p < 0,0001) relataram frequentá-la. Em relação ao ambiente utilizado para o estudo, 87,4\% (299/342, p < 0,0001) dos respondentes informaram possuir ambiente fixo. 
A autogestão do tempo de estudo é fundamental para a construção ativa do conhecimento ${ }^{16,17}$. Investigou-se a periodicidade semanal de estudos, sendo que 72,2\% (247/342; $\mathrm{p}<$ 0,0001 ) revelaram estudar mais de três vezes por semana.

A partir da década de 1980, houve a massificação e popularização do uso de computadores, com tecnologia e inovações em apoio ao processo educacional ${ }^{18}$. Em relação às ferramentas eletrônicas próprias, como telefone celular com acesso à internet, tablets, computador, etc., apenas dois discentes $(0,6 \%, p<0,0001)$ responderam não possuir ao menos um desses equipamentos, corroborando o conceito de que as tecnologias são importantes instrumentos na educação ${ }^{19}$. Estudos mostram que a internet e recursos eletrônicos - como livros-texto eletrônicos, coleções de livros didáticos ou enciclopédias eletrônicas - são os mais frequentemente utilizados por alunos de Medicina ${ }^{20,21,22}$. Assim, a metodologia de ABP requer grande número de computadores na IES para alunos que não possuam tais ferramentas ${ }^{6}$.

Atualmente, as informações são facilmente adquiridas por meio da internet, revistas eletrônicas e base de dados de artigos facilmente disponíveis, e estudos atuais mostram a internet como o recurso mais utilizado por alunos de Medicina $^{20,21}$. Quanto à distribuição das respostas de acordo com a frequência indicada pelos alunos, sobre o uso da internet como complemento do estudo, nota-se que a maioria dos alunos (149/342, 43,6\%) respondeu fazê-lo com muita frequência, e a minoria $(20 / 342,5,8 \%)$ relatou ser esta uma prática "pouco frequente" ( $\mathrm{p}<0,0001)$. Portanto, estes alunos se encaixam no perfil desta geração, que conhece estas ferramentas de busca de informação e tem facilidade em seu uso.

Os objetos digitais de aprendizagem podem auxiliar os processos educacionais no ambiente escolar, e as vídeoaulas são capazes de integrar múltiplas mídias e linguagens, com códigos e significações predominantemente audiovisuais. Bonini-Rocha et al. ${ }^{18}$ compararam 141 alunos da disciplina de Anatomia Humana em relação a satisfação, percepção de aprendizagem e desempenho em videoaulas e aulas expositivas, concluindo que ambas foram adequadas, promovendo o aprendizado do tema, apesar de somente ter sido avaliado a memória de curto prazo. Os discentes também foram questionados sobre a frequência habitual de acesso a videoaulas para complementar os estudos. A maioria dos respondentes (149/342, 43,6\%) revelou recorrer com pouca frequência a este recurso, enquanto a minoria ou nunca o fez $(29 / 342,8,4 \%)$ ou o faz sempre $(20 / 342,5,8 \%)^{18}$. Jou e Sperb ${ }^{23}$ relatam que a proficiência em leitura - isto é, quanto maior o hábito da leitura, maior será a capacidade de compreensão - e talvez ler mais seriam um treinamento para melhorar a aprendizagem ${ }^{23}$.

\section{Domínio "estratégias de planejamento e organização do estudo"}

Em relação aos constructos do domínio "estratégias de planejamento e organização do estudo", observou-se que ao menos 50\% dos participantes acham fácil cumprir um horário de estudo, evitam situações que poderiam desviar sua atenção e iniciam o estudo para o fechamento da tutoria no mesmo dia da abertura do problema em "cerca de 50\% das vezes" e que, para até $75 \%$ deles (257 alunos), isso ocorre no máximo "com muita frequência". Quanto a estudar sozinho, ter foco nos objetivos específicos de aprendizagem e estudar para todos os objetivos, 50\% dos alunos responderam que o fazem "com muito frequência" a "sempre", e metade dos alunos relatou estudar sozinho com muita frequência.

A maioria dos alunos estudados encontra-se na faixa etária da geração $Z$, chamada de "geração silenciosa", multiconectada com fones de ouvidos e que nunca conheceu o mundo

TABELA 1

Estatística descritiva das facetas do domínio "estratégias de planejamento e organização do estudo". Cesupa, 2017 Domínio e facetas

Mediana (DIQ) Mín. - Máx.

P25/P75

Estratégias de planejamento e organização do estudo

1. Acho fácil cumprir um horário de estudo.

$50(50)$

$75(25)$

$50(25)$

$50(50)$

$75(25)$

$75(25)$

$25(0)$

$0-100$

$25 / 75$

(equipamentos eletrônicos, conversas paralelas, aplicativos de bate-papo, etc.).

4. Inicio meus estudos para o fechamento da tutoria no mesmo dia da abertura do problema.

5. Ao iniciar o estudo, tenho foco nos objetivos específicos de aprendizagem.

6. Estudo para todos os objetivos propostos.

7. Quando estudo para o fechamento do problema utilizando a internet, perco-me em detalhes e não consigo me lembrar das ideias principais.

DIQ: desvio interquartílico. Mín.: mínimo. Máx.: máximo. P25/P75: percentis 25 e 75, respectivamente.

Fonte: Protocolo de pesquisa, 2017.

$0-100 \quad 75 / 100$

$0-100 \quad 50 / 75$

$0-100 \quad 25 / 75$

$25-100 \quad 75 / 100$

$25-100 \quad 75 / 100$

$0-100 \quad 25 / 25$


sem computadores pessoais, telefones celulares, sistemas de jogos, leitores de MP3 e internet ${ }^{9}$. A capacidade de fazer muitas coisas ao mesmo tempo seria por meio da automatização, defendida por Gagné, utilizando o mínimo de consciência ${ }^{10}$. Mesmo sendo multiconectados, esses estudantes conseguem ter concentração ao estudar.

O estudo individual é importante na $\mathrm{ABP}$, sem comunicação com o grupo, justamente para que a quantidade de informações pesquisadas seja consideravelmente consistente e variada para resolver o problema ${ }^{24}$. A importância da discussão em grupo durante o fechamento de uma sessão tutorial demonstra a diversidade de informações entre os alunos no grupo para complementar o estudo e potencializar o aprendizado ${ }^{25}$. Ao serem questionados sobre a organização do estudo quando utilizam a internet nas pesquisas, até $75 \%(257 / 342)$ dos estudantes responderam que se perdem em detalhes e não conseguem se lembrar das ideias principais entre "nunca" e "raramente"" (Tabela 1). Esta geração, apesar de conectada à internet e da facilidade de acesso às informações por meio de links, muitas vezes acaba não retornando ao assunto principal inicial da sua pesquisa ${ }^{4}$.

\section{Domínio "estratégias de aprendizagem"}

No que diz respeito às estratégias de aprendizagem, observa-se que o uso do mapa conceitual elaborado na abertura não é frequente, pois para até $25 \%$ dos alunos isso nunca acontece, e até $75 \%$ deles responderam que isso ocorre apenas "raramente" (Tabela 2).

O mapa conceitual é uma estrutura esquemática para representar um conjunto de conceitos imersos numa rede de proposições e é uma estratégia potencialmente facilitadora de uma aprendizagem significativa ${ }^{26,27}$. Alunos que utilizam o mapa conceitual, que tem três elementos fundamentais - os conceitos, a relação entre eles e o propósito -, conseguem fazer conexões entre os conhecimentos adquiridos e os preexisten- tes, integrando as ciências básicas com a clínica, promovendo pensamento crítico, além de auxiliar na formação de hipóteses, com interrelações entre os conceitos que não estavam tão claros, em relação a alunos que não utilizaram o mapa ${ }^{28,29,30}$. Provavelmente, o mapa conceitual seria pouco utilizado por falta de alcance pelos discentes do seu real significado, sendo esta lacuna uma responsabilidade também do professor-tutor.

A busca dos termos de estudo no idioma inglês acontece apenas em $50 \%$ das vezes para a maioria dos estudantes (75\%, 257/342), sugerindo que esta é outra estratégia pouco utilizada, embora uma das competências dos egressos seja dominar língua estrangeira, de preferência a língua franca, para se manter atualizado com os avanços da medicina ${ }^{5}$ (Tabela 2).

Já a síntese do estudo em forma de resumo é feita "sempre" por no mínimo $25 \%$ dos alunos e "com muita frequência" por $50 \%$ deles, e a elaboração de esquemas, mapas conceituais etc. é referida por até 75\% dos alunos como "muito frequente" (Tabela 2).

Jou e Sperb ${ }^{23}$ mostram que alunos que resumem o texto estudado têm melhor desempenho do que aqueles que não o fazem, especialmente se o resumo for feito após um tempo, dias depois.

A contextualização ocorre entre "raramente" e "sempre" para todos os estudantes, sendo que no mínimo 75\% dos alunos relataram que tentam imaginar de que forma o que estão estudando se aplica à sua vida diária pelo menos aproximadamente $50 \%$ das vezes em que estudam (Tabela 2).

O contato inicial entre o estudante e a comunidade é importante, favorecendo a contextualização da teoria na prática vivenciada, já que há grande dificuldade de correlacionar o exagerado volume de informações teóricas recebidas. Além disso, o aluno tem que ter motivação para aprender e, para isso, deve ter interesse no que está fazendo, ter significado pessoal para levar a uma aprendizagem significativa ${ }^{3,17,24,25,31,32}$.

\section{TABELA 2}

Estatística descritiva das facetas do domínio "estratégias de aprendizagem". Cesupa, 2017

Domínio e facetas

Mediana (DIQ) Mín. - Máx.

P25/P75

Estratégias de aprendizagem

1. Quando estou estudando, consulto o mapa conceitual elaborado na tutoria de abertura.

2. Quando utilizo a internet para estudar, faço busca utilizando os termos em INGLÊS.

3. Quando estou estudando, sintetizo em forma de texto o que estou lendo, com minhas próprias palavras (próprio resumo).

4. Quando estou estudando, faço desenhos, mapas conceituais ou esquemas para me ajudar a entender o que estou estudando.

5. Quando estou estudando, tento ver de que forma aquilo pode aplicar-se à minha vida diária (contextualização).

DIQ: desvio interquartílico. Mín.: mínimo. Máx.: máximo. P25/P75: percentis 25 e 75, respectivamente.

Fonte: Protocolo de pesquisa, 2017.
25 (25)

$25(25)$

$\begin{array}{lcc}25(25) & 0-100 & 0 / 25 \\ 25(25) & 0-100 & 25 / 50 \\ 75(50) & 0-100 & 50 / 100 \\ 75(25) & 0-100 & 50 / 75 \\ 75(25) & 25-100 & 50 / 75\end{array}$


Domínios "qualidade das informações" e "autopercepção da retenção do conhecimento"

Os alunos de metodologias ativas têm maior variedade e melhor qualidade das fontes bibliográficas de estudos, mostrando-se capazes de ter escolhas pessoais do seu melhor recur$\mathrm{so}^{3,33,34,35}$. Rankin ${ }^{36}$ já referia que, na $\mathrm{ABP}$, os estudantes gastam mais tempo na biblioteca e usam uma grande variedade de recursos para obter informações, utilizando de três a seis recursos para a resolução do problema.

Avaliando os resultados, é possível notar que para no mínimo $25 \%$ dos estudantes a utilização de mais de um livro-texto em seus estudos e o acesso a artigos por meio de plataformas de bases de dados científicos ocorre "sempre", sendo que $50 \%$ dos alunos ofereceram respostas a estas questões que variaram de "nunca" a "muito frequente".

Já em relação ao uso de apostilas comerciais e resumos não próprios, até $75 \%$ dos alunos responderam que isso ocorre em aproximadamente " $50 \%$ das vezes" (Tabela 3), dado que é preocupante, pois essas fontes podem não ser de origem segura. Shankar e Nandy ${ }^{37}$ avaliaram alunos do curso de Medicina em Aruba e encontraram maior utilização da busca de informação em resumos de estudos $(84,3 \%)$, seguidos de livros-texto $(80,4 \%)$, com pouco uso de internet $(35,3 \%)$ e revistas médicas $(15,7 \%)$.

Saparova e Nolan ${ }^{22}$, embora teham avaliado um número pequeno de alunos, analisaram recursos de informações eletrônicas, revelando que o aprendizado dos alunos que estudavam na Wikipédia não foi inferior ao dos que estudavam nos outros recursos, como UpToDate e Acess Medicine, talvez em virtude da organização do estudo pelos alunos e da ampla base de informações. Fatores positivos do uso da Wikipédia, segundo os alunos, seriam a conexão com links favorecendo a navegação e escrita de fácil entendimento para o público geral. Fatores negativos no aprendizado seriam palavras difíceis, não familiares e complexidade visual. Desta maneira, se aconselha que educadores e bibliotecários auxiliem na seleção e no uso de textos clássicos com novos recursos digitais ${ }^{22}$.

Musal et al. ${ }^{34}$ e Luna e Bernardes ${ }^{25}$ evidenciaram que, quanto mais os alunos se aprofundavam no estudo em diferentes recursos, como fontes seguras e evidências científicas, embora não seja estatisticamente significativo, mais isto afetava negativamente a profundidade da discussão e a crítica. Talvez isso ocorra por falta de uma boa estruturação da discussão, já que foi observado mais no primeiro ano do que no terceiro ano do curso.

As capacidades individuais de autoaprendizagem e busca do conhecimento são destaques nos cursos de ABP, como também saber lidar com incertezas e limites pessoais, adquirindo progressivamente autonomia para aprender e melhorar a confiança ${ }^{38}$.

Quanto ao último domínio avaliado, para 50\% dos estudantes a resposta aos dois itens referentes à autopercepção da retenção do conhecimento variou entre "cerca de 50\% das vezes" e com "muita frequência". Um aluno (0,3\%) respondeu que "nunca" tem a certeza de ter adquirido o conhecimento relativo aos objetivos de aprendizagem propostos após finalizar o estudo; e quatro alunos (1,2\%) responderam que "nunca" têm facilidade em resgatar o conhecimento ou as informações apreendidas em módulos anteriores quando determinado assunto é abordado novamente (Tabela 3).

Toledo Junior et al. ${ }^{33}$ relataram que alunos de metodologias ativas têm maior retenção dos conhecimentos e aprendi-

\begin{tabular}{|c|c|c|c|}
\hline \multicolumn{4}{|c|}{$\begin{array}{l}\text { Estatística descritiva das facetas dos domínios "qualidade das informações" } \\
\text { e "autopercepção da retenção do conhecimento". Cesupa, } 2017\end{array}$} \\
\hline Domínios e facetas & Mediana (DIQ) & Mín. - Máx. & P25/P75 \\
\hline \multicolumn{4}{|l|}{ Qualidade das informações } \\
\hline 1. Utilizo mais de um livro-texto em meus estudos (referenciado ou não). & $75(25)$ & $0-100$ & $75 / 100$ \\
\hline 2. Estudo apenas por apostilas comerciais (físicas ou on line) e/ou resumos não próprios. & $25(25)$ & $0-100$ & $25 / 50$ \\
\hline $\begin{array}{l}\text { 3. Quando utilizo a internet para estudar, procuro acessar artigos científicos, a exemplo dos } \\
\text { disponíveis nas plataformas EBSCO, Pubmed/Medline, SciELO, Lilacs, etc. }\end{array}$ & $75(50)$ & $0-100$ & $50 / 100$ \\
\hline \multicolumn{4}{|l|}{ Autopercepção da retenção do conhecimento } \\
\hline $\begin{array}{l}\text { 1. Quando finalizo o estudo, tenho a certeza de ter adquirido o conhecimento relativo aos } \\
\text { objetivos de aprendizagem propostos. }\end{array}$ & $50(25)$ & $0-100$ & $50 / 75$ \\
\hline $\begin{array}{l}\text { 2. Sempre que determinado assunto é abordado novamente, porém em outro módulo, tenho } \\
\text { facilidade em resgatar o conhecimento ou as informações apreendidas em módulos } \\
\text { anteriores. }\end{array}$ & $50(25)$ & $0-100$ & $50 / 75$ \\
\hline
\end{tabular}

DIQ: desvio interquartílico. Mín.: mínimo. Máx.: máximo. P25/P75: percentis 25 e 75, respectivamente.

Fonte: Protocolo de pesquisa, 2017. 
zado. E que o resgate do conhecimento é extremamente importante para a construção de aprendizagens significativas ${ }^{33,39}$.

O domínio "qualidade das informações", que compreendeu a confiabilidade das fontes pesquisadas e variou de 0 a 100, apresentou escore mediano significativamente maior que os demais $(\mathrm{Md}=75)$, o que pode ser interpretado como o domínio em que os estudantes apresentaram melhor desempenho, ou seja, 50\% dos estudantes utilizavam os melhores recursos de aprendizagem no mínimo com muita frequência ( $p<0,0001$ ). Em seguida, tem-se o domínio "estratégias de planejamento e organização do estudo", cujo valor mediano foi igual a 67,9 , demonstrando que a capacidade do aluno para elaborar e seguir uma rotina que lhe garanta um ambiente favorável ao estudo se apresenta como a melhor estratégia em quase $70 \%$ das vezes. Quanto à autopercepção da retenção do conhecimento, os alunos consideraram que assimilavam os conteúdos trabalhados nas sessões tutoriais em mais de $60 \%$ das vezes $(\mathrm{Md}=62,5)(\mathrm{p}<0,0001)$. No entanto, o domínio "estratégias de aprendizagem" apresentou o menor escore mediano ( $\mathrm{Md}=50)$ quando comparado aos outros domínios, significando que os estudantes utilizavam as melhores estratégias para otimizar o tempo e garantir memorização eficiente em apenas cerca de $50 \%$ das vezes em que estudavam, de forma que ele estava aquém dos demais ( $\mathrm{p}<0,0001)$.

A Tabela 4 apresenta a matriz de correlação efetuada entre os domínios investigados. Todos os domínios apresentaram correlações positivas e moderadas entre si, variando de $\mathrm{r}=0,1523$ (qualidade das informações versus autopercepção da retenção do conhecimento) a $r=0,4123$ (estratégias de planejamento e organização versus autopercepção da retenção do

Figura 2

Comparação dos escores ${ }^{a}$ obtidos no estudo segundo o domínio. Cesupa, 2017

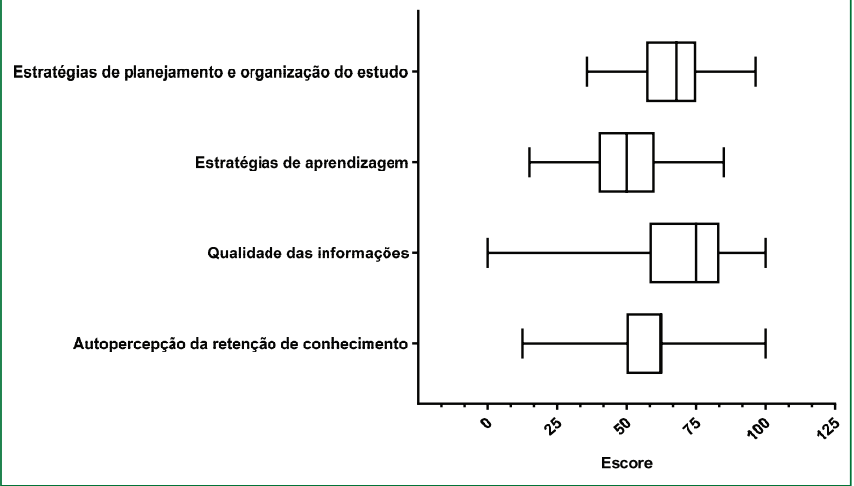

$\mathrm{p}<0,0001$ † (Análise de Variância de Friedman). † Estatisticamente significativo. a Expressos como mediana, percentis 25 e 75, e escores mínimo e máximo. Fonte: Protocolo de pesquisa, 2017 conhecimento) ( $\mathrm{p}<0,0001)$. Isto sugere que, quanto melhor a estratégia de planejamento e organização, maior a autopercepção da retenção do conhecimento, assim como estratégias de aprendizagem bem definidas e informações de qualidade também se refletem em maior percepção da retenção do próprio conhecimento.

\begin{tabular}{|c|c|c|c|c|}
\hline \multicolumn{5}{|c|}{$\begin{array}{c}\text { TAвELA } 4 \\
\text { Correlações entre os domínios abordados } \\
\text { pelo questionário. Cesupa, } 2017\end{array}$} \\
\hline & $\begin{array}{l}\text { Estratégias de } \\
\text { planejamento/ } \\
\text { organização }\end{array}$ & $\begin{array}{l}\text { Estratégias de } \\
\text { aprendizagem }\end{array}$ & $\begin{array}{l}\text { Qualidade } \\
\text { das } \\
\text { informações }\end{array}$ & $\begin{array}{l}\text { Autopercepção } \\
\text { da retenção do } \\
\text { conhecimento }\end{array}$ \\
\hline $\begin{array}{l}\text { Estratégias de } \\
\text { planejamento/ } \\
\text { organização }\end{array}$ & 1,0000 & & & \\
\hline $\begin{array}{l}\text { Estratégias de } \\
\text { aprendizagem }\end{array}$ & 0,3453 & 1,0000 & & \\
\hline $\begin{array}{l}\text { Qualidade das } \\
\text { informações }\end{array}$ & 0,2413 & 0,2231 & 1,0000 & \\
\hline $\begin{array}{l}\text { Autopercepção } \\
\text { da retenção do } \\
\text { conhecimento }\end{array}$ & 0,4123 & 0,3210 & 0,1523 & 1,0000 \\
\hline
\end{tabular}

$\mathrm{p}<0,0001^{\dagger}$ (Matriz de correlação). ${ }^{\dagger}$ Estatisticamente significativo. Fonte: Protocolo de pesquisa, 2017.

A correlação positiva existente entre "estratégias de planejamento e organização" e os demais domínios também pode revelar que o hábito de planejar o estudo está positivamente associado às melhores estratégias de aprendizagem e à busca por fontes de melhor qualidade técnica, resultando em maior percepção da retenção do conhecimento pelo aluno.

Comparando-se os escores relacionados aos domínios "estratégias de planejamento e organização do estudo", "estratégias de aprendizagem", "qualidade das informações" segundo a turma, verificou-se que a turma MD8 apresentou escore mediano inferior ao das demais. $\mathrm{O}$ oitavo semestre do Cesupa é o último semestre de sessões tutoriais. Os baixos valores medianos deste grupo de estudantes talvez se devam à perda de interesse no estudo autodirigido ou ao "cansaço" de tutorias, embora valores medianos de autopercepção de retenção do conhecimento não tenham sido inferiores aos das outras turmas, além de não terem sido avaliados os rendimentos dessa turma.

A maioria dos estudantes na metodologia ativa promove o estudo autodirigido e adquire esta habilidade com maturidade já no primeiro ano de estudo, mas é importante o suporte - como planejamento e organização - para aprimorar esta habilidade ${ }^{30,36,40}$. Preeti et al. ${ }^{41}$ avaliaram 72 estudantes de Medicina na metodologia $\mathrm{ABP}$, sendo que a grande maioria $(91,6 \%)$ relatou ser estimulada ao estudo autodirigido ${ }^{41}$. O mesmo foi observado no estudo de Carvalho ${ }^{42}$, que mostrou 
um valor elevado na Escala Jefferson de Aprendizagem ao Longo da Vida em estudantes de Medicina em Santarém (PA), o que pode ser considerado uma consequência desta nova metodologia de ensino, que estimularia o autoaprendizado.

$\mathrm{O}$ estudo de Musal et al. ${ }^{34}$ mostrou que alunos turcos do terceiro ano do curso de Medicina usam mais livros-texto $(44,4 \%)$ do que os do primeiro ano $(24,3)$, pela qualidade na busca de informação. Já as anotações de aulas (apostilas) foram frequentemente usadas por ambas as turmas, por apresentarem conteúdo das aulas de maneira resumida $(95,7 \%$ do primeiro ano e $95,8 \%$ do terceiro ano $)^{34}$.

No estudo, no ambiente acadêmico, à medida que os alunos adquirem mais experiência, podem fazer melhor uso do tempo de estudo, sabendo selecionar a qualidade dos tópicos estudados ${ }^{23}$.

Smolka et al. ${ }^{7}$, em estudo que avaliou a percepção dos estudantes de Medicina acerca da ABP, relataram de maneira enfática que a capacidade de aprender a aprender é desenvolvida de forma crescente ao longo do curso. Mudanças de comportamento e atitudes também são características dos estudantes na $\mathrm{ABP}$, que criam hábitos de estudos com o passar do tempo, como ida à biblioteca e procura de fontes de estudo, e, quanto mais avançam no curso, mais adquirem a capacidade de focar e selecionar o que devem estudar ${ }^{3}$. Também Luna e Bernardes ${ }^{25}$ apontaram que os acadêmicos, com o passar do tempo, melhoram a sua objetividade, a busca de fontes seguras e a humildade para reconhecer as próprias limitações. Mesmo assim, nossos alunos já mostram maturidade e responsabilidade desde o primeiro ano do curso para iniciar o estudo autodirigido. Entretanto, Fasce et al. ${ }^{33}$ e Mahmud et al..$^{44}$ enfatizam a importância do esforço pessoal para obter maior controle da aprendizagem ${ }^{43,44}$.

\section{CONCLUSÃO}

Avaliando-se as estratégias de autoaprendizagem utilizadas, conclui-se que a grande maioria dos discentes estudados utiliza as novas tecnologias de informação e comunicação, sendo a internet o principal meio de pesquisa dos objetivos. No entanto, não costumam usar as videoaulas como complementação do estudo. Além disso, otimizam seu tempo e frequentam lugares fixos, como a biblioteca. Os alunos planejam e cumprem seu horário de estudo individualmente e, mesmo multiconectados, não perdem o foco e a concentração na busca de todos os objetivos da sessão tutorial.

A qualidade das informações utilizadas pelos estudantes foi adequada, e estas foram buscadas por meio de mais de um livro-texto e artigos científicos hospedados em plataformas confiáveis. Entretanto, muitos alunos ainda utilizam resumos ou apostilas não próprias para seu estudo. Nas estratégias de aprendizagem, o mapa conceitual é pouco empregado, e a execução de resumos e esquemas próprios poderia ser mais bem aproveitada. A busca de artigos científicos em língua inglesa foi pouco usada, ao passo que a contextualização foi uma das estratégias bem aplicadas.

A maioria dos alunos refere ter adquirido o conhecimento dos objetivos de aprendizagem e resgatado as ideias em momentos posteriores, mostrando capacidade de autopercepção na retenção do conhecimento por meio do estudo autodirigido. Isto está diretamente relacionado às melhores estratégias de planejamento, organização e aprendizagem, assim como à melhor qualidade das informações. Não houve diferença nos domínios pesquisados em relação às diferentes turmas, exceto o oitavo período. Para otimizar o desempenho do estudante, sugere-se um guia de orientações de autoaprendizagem.

\section{REFERÊNCIAS}

1. Augustin M. How to learn effectively in medical school. YALE Journal of Biologyand Medicine. 2014; 87: 207-212.

2. Romanelli F. Learning Styles: A Review of Theory, Application, and Best Practices. American Journal of Pharmaceutical Education. 2009; 73 (1): Article 9.

3. Cezar PHN, Guimarães FT, Gomes AP, Rõças G, Siqueira-Batista R. Transição Paradigmática na Educação Médica: Um Olhar Construtivista Dirigido à Aprendizagem Baseada em Problemas. Rev. Bras. Educ. Med 2010; 34 (2): 298-303.

4. Gomes AP, Rego S. Transformação da Educação Médica: É possível formar um Novo Médico a partir de Mudanças no Método de Ensino-Aprendizagem? Rev. Bras. Educ. Med. 2011; 35(4): 557-566.

5. Brasil. Conselho Nacional de Educação, Câmara de Educação Superior. Resolução CNE/ CES NNo 3 de 20 de junho de 2014. Diretrizes Curriculares Nacionais do Curso de Graduação em Medicina. Disponível em: <http://portal. mec.gov.br/index.php?option=com_docman\&task=doc_ download\&gid=15874\&Itemid $>$. Acesso em: 17 de dezembro 2015.

6. Centro Universitário do Estado do Pará (CESUPA). Projeto Pedagógico do Curso de Medicina- Belém. 297p. (Série Material Instrucional do CESUPA, 2016). Disponível em: <http:/ / www.cesupa.br/Graduacao/Biologicas/docs / PROJETO_PEDAG\%C3\%93GICO_MEDICINA_CESUPA. pdf>. Acesso em: 03 de julho de 2017.

7. Smolka MLRM, Gomes AP, Siqueira-Batista R. Autonomia no Contexto Pedagógico: Percepção de Estudantes de Medicina acerca da Aprendizagem Baseada em Problemas. Rev. Bras. Educ. Med 2014; 38(1) 5-14. 
8. Towle A, Cottrell D. Self directed learning. Arch. Dis. Child. 1996;74: 357-359.

9. Leitinho MC, Sá Carneiro CCB. Aprendizagem baseada em problemas: uma abordagem pedagógica e curricular. In: Veiga IPA, Amaral AL, Dalben AILdeF, Sá Carneiro CCB, Romanowski JP, Araujo JCS, Leitinho MC, Martins PLO. (Org.) Novas Tramas para as Técnicas de Ensino e Estudo. Ed. Papirus. 2013.

10. Boruchovitch E. Estratégias de aprendizagem e desempenho escolar: considerações para a prática educacional. Psicologia Reflexão e Crítica. Rev. de la Universidad Federal do Rio Grande do Sul.1999; 12(2).

11. Bartalo L, Guimarães SER. Estratégias de estudo e aprendizagem de alunos universitários: um estudo exploratório. Informação \& Informação, Londrina 2008;13(2) 1-14.

12. Boninger M. Scholarly Concentrations in the Medical Student Curriculum. Acad Med 2010; 85(3).

13. Olaussen BS, Braten I. Identifying latent variables measured by the Learning and Study Strategies Inventory (LASSI) in norwegian college students. The Journal of Experimental Education, Washington 1998;67(1)82-96.

14. Bartalo L. Mensuração de estratégias de estudo e aprendizado de alunos universitários: learning and study strategies inventory (LASSI) adaptação e validação para o Brasil. Tese (Doutorado em Educação) - Universidade Estadual Paulista Julio de Mesquita Filho, Marília. 2006, $215 \mathrm{f}$.

15. Ayres M, Ayres Júnior M, Ayres D L, Santos A. BioEstat 5.45. Aplicações Estatísticas nas Áreas das Ciências Biológicas e Médicas: Belém: MCT; IDSM; CNPq, 2015. 364 p. il. Acompanha CD-ROM.

16. Wood DF. ABC of learning and teaching in medicine. Problem based learning. BMJ. 2003; 326(8).

17. Holanda VRde, Pinheiro AKB, Pagliucall LF. Aprendizagem na educação online: análise de conceito. Rev Bras Enfermagem 2013; 66(3) 406-11.

18. Bonini-Rocha AC, Oliveira LF de, Rosat RM, Ribeiro MFM. Satisfação, Percepção de Aprendizagem e Desempenho em Vídeoaula e Aula Expositiva. Ciências \& Cognição 2014; 19(1) 47-57.

19. Bransford JD, Brown AL, Cocking RR. How people learn: Brain, Mind, Experience and School. Expanded Edition. Washington, DC, USA. National Academies Press, 2000.

20. Al-Dress AA. et al. Students' perception towards the problem based learning tutorial session in a system-based hybrid curriculum. Saudi Med J. 2015; 36(3) 341.

21. Ruiz-Gallardo JR, Gonzalez-Geraldo JL, Castaño S. What are our students doing? Workload, time allocation and time management in PBL instruction. A case study in Science Education. Teaching and Teacher Education 2016; 53, p. 51-62.

22. Saparova DMA, Nolan NS. Evaluating the appropriateness of electronic information resources for learning. Journal of Medical Library Association 2016;104(1) January.

23. Jou G, Sperb TM. A metacognição como estratégia reguladora de aprendizagem. Psicologia: Reflexão e Crítica 2006;19(2)177-185.

24. Farias PAM, Martin A LdeA, Cristo CS. Aprendizagem Ativa na Educação em Saúde: Percurso Histórico e Aplicações. Rev. Bras. Educ. Med 2015;39(1)143- 158.

25. Luna WF, Bernardes JS. Tutoria como Estratégia para Aprendizagem Significativa do Estudante de Medicina. Rev. Bras. Educ. Med 2016;40(4) 653-662

26. Ruiz-Moreno L, Sonzogno MC, Silva Batista SH da, Alves Batista N. Mapa conceitual: ensaiando critérios de análise. Ciência \& Educação 2007;13(3)453-463.

27. Tavares R. Construindo mapas conceituais. Ciências \& Cognição 2007;12, p.72-85.

28. Gorbaneff Y, Cancino A. Mapa conceptual para el aprendizaje basado en problemas. Estudios Gerenciales 2009;25(110)111-124, enero-marzo.

29. Veiga IPA. Ensinar, Aprender, Pesquisar e avaliar com mapas conceituais. In: Veiga IPA, Amaral AL, Dalben AILde F, Sá Carneiro, CCB, Romanowski JP, Araujo JCS, Leitinho O MC, Martins PLO. Novas Tramas para as Técnicas de Ensino e Estudo. Ed. Papirus. 2013.

30. Leon LBde,Onófrio FdeQ. Aprendizagem Baseada em Problemas na Garduação Médica - Uma Revisão da Literatura Atual. Rev. Bras. Educ. Med 2015; 39(4) 614-619.

31. Gomes AP, Rego S. Paulo Freire: Contribuindo para pensar mudanças de estratégias no ensino de Medicina. Rev. Bras. Educ. Med 2014;38(3) 293-313

32. Lobo LC. Educação Médica nos Tempos Modernos Rev. Bras. Educ. Med 2015; 39(2)328-332.

33. Toledo Junior AC de C, Ibiapina C da C, Lopes SCF, Rodrigues ACP, Soares SMS. Aprendizagem baseada em problemas: uma nova referência para a construção do currículo médico. Rev Med Minas Gerais 2008;18(2)123-131.

34. Musal B, Gursel Y, Tashiran HC, Ozan S, Tuna A. Perceptions of first and trird year medical students on self-study and reporting processes os problem-based learning. BCM Med Educ 2001; 4(16)1-7.

35. Murad MH, Coto-Yglesias F, Varkey P, Prokop LJ, Murad A L. The effectiveness of self-directed learning in health professions education: a systematic review. Med. Educ. 2010; 44, p.1057-1068. 
36. Rankin JA. Problem-based medical education: effect on library use. Bull Assoc Med Libr 1992 ;80(1), January.

37. Shankar PR, Nandy A. Student feedback on problem-based learning process. AMJ 2014; 7(12) 522.

38. Gomes R, Brino RdeF, Aquilante AG, AvóLRdaS. Aprendizagem Baseada em Problemas na formação médica e o currículo tradicional de Medicina: uma revisão bibliográfica. Rev. Bras. Educ. Med 2009;33(3) 444-451.

39. Batista NA, Batista SHS da S. A prática como eixo da aprendizagem na graduação médica. In: Puccini RF, Sampaio LO, Batista NA. A formação médica na UNIFESP: excelência e compromisso social [online]. São Paulo: Editora Unifesp, 2008. P.101-115. ISBN 978-85-61673-66-6. Available from Scielo Books http:/ / books.scielo.org.

40. Soliman M, Al-ShaIlkh G. Readiness for self-directed learning among First Year Saudi Medical students: A descriptive study. Pak J Med Sci 2015 ;31(4)799-802

41. Preeti B, Ashish A, Shriram G. Problem Based Learning (PBL) - An Effective Approach it Improve Learning Outcomes in Medical Teaching. Journal of Clinical and Diagnotic Research JCDR 2013; 7(12) 2896-2897.

42. Carvalho CAS de. Análise de estratégias e habilidades de aprendizagem ao longo da vida em estudantes de medicina de um curso da amazônia brasileira. 2016. Tese (Mestrado profissional em ensino em saúde na amazônia). Belém: Universidade do Estado do Pará.

43. Fasce E, Perez C, Ortiz L, Parra P, Ibañez P, Matus O. Aprendizaje autodirigido y su relacion con el perfil valórico en estudiantes de medicina. Rev. Med. Chile. 2013; $141,15-22$

44. Mahmud W, et al. Self-directed learning and research attitudes among medical students. JCPSP Journal of the College of Physicians and Surgeons- Pakistan 2014; 24(3)173177.

\section{CONTRIBUIÇÃO DOS AUTORES:}

Irma Douglas Paes Barreto: idealizadora do estudo e responsável por toda coleta dos dados e redação do texto;

Ismari Perini Furlaneto: contribuiu com o desenho do estudo, análise e interpretação dos dados;

Patrick Abdala Fonseca Gomes e Bruno Acatauassu Paes Barreto: contribuíram com o desenho do estudo, análise da redação e revisão final do texto;

\section{CONFLITO DE INTERESSES:}

Não existe conflito de interesses.

\section{ENDEREÇO PARA CORRESPONDÊNCIA:}

Endereço: Avenida Pedro Alvares Cabral 264 - Ap 2401 Bairro Umarizal - Belém (PA) - CEP: 66050-400;

email: irmacdouglas@gmail.com 